\title{
LINEAR ALGEBRA AND TOPOLOGY
}

\author{
BY SYLVAIN E. CAPPELL ${ }^{1}$ AND JULIUS L. SHANESON 1
}

Two real entried (respectively: orthogonal) $n \times n$ matrices $A$ and $B$ are said to be linearly similar if there is an invertible real (resp. orthogonal) $n \times n$ matrix $C$ with $C A C^{-1}=B$. Of course, $A, B$ and $C$ may be regarded as linear functions from $R^{n}$ (resp. isometries from $S^{n-1}$ ) to itself. The matrices $A$ and $B$ are said to be (resp. homogeneously differentiably) differentiably similar if there is a diffeomorphism $f: R^{n} \rightarrow R^{n}$, with ${ }^{2} f(0)=0$ (resp. $f: S^{n-1} \rightarrow$ $S^{n-1}$ ) for which $f A f^{-1}=B$. Differentiating this equation at 0 , one sees immediately that differentiable similarity on $R^{n}$ is the same as linear similarity with $C=(D f)_{(0)}$. A celebrated theorem of de Rham [7], [8] proves the corresponding equivalence for homogeneous differential similarity of orthogonal transformations of $S^{n-1}$.

The matrices $A$ and $B$ are said to be (resp. homogeneously) topologically similar if there is a homeomorphism $f: R^{n} \rightarrow R^{n}$ with $^{2} f(0)=0$ (resp. $f$ : $S^{n-1} \rightarrow S^{n-1}$ ) and $f A f^{-1}=B$. For $S^{1}$, Poincaré showed, by defining rotation numbers, the equivalence of topological and linear similarity there. The study of the analogous question on $S^{n-1}$ was continued by de Rham. In studying when topological similarity would imply linear similarity on $R^{n}$, Kuiper and Robbin [5] showed that the general problem could be reduced to the following conjecture.

Topological Characterization Conjecture. If $A$ and $B$ are matrices whose eigenvalues (real or complex) have modulus 1, then topological similarity is equivalent to linear similarity.

This conjecture applied, for example, to all orthogonal matrices. They proved the conjecture for matrices whose eigenvalues are not roots of unity. In fact they showed that the general conjecture reduced to the case when $A$ and $B$ were periodic matrices, i.e. $A^{n}=I$, for some $n$. Thus, they made the conjecture:

CONJECTURE: If $A$ and $B$ satisfy $A^{n}=I=B^{n}$, then topological similarity is equivalent to linear similarity.

Received by the editors February 27, 1979.

AMS (MOS) subject classifications (1970). Primary 57E05, 57A15, 15A21; Secondary 54C05, 12A50, 34D05, 22A05, $15 \mathrm{~A} 18$.

1 This work was partially supported by NSF Grants.

2 If the condition $f(0)=0$ is dropped, the equivalence classes are trivially seen to be the same. 
They showed this for $n=1,2,3,4,6$. It is also valid if all the eigenvalues of $A$ are primitive $n$th roots of unity. Results obtained independently on this problem by Dennis Sullivan and Reinhardt Schultz [9], using classifying space methods, showed it for $n=p^{s}$ and $n=2 p^{s}, p$ an odd prime, and perhaps some more cases.

We announce the following.

Counterexamples to the Conjectures. The orthogonal matrices $A_{+1}$ and $A_{-1}$ where

$$
A_{\epsilon}=\left[\begin{array}{cccccc}
\frac{\epsilon}{\sqrt{2}} \frac{\epsilon}{\sqrt{2}} & & & \\
\frac{-\epsilon}{\sqrt{2}} \frac{\epsilon}{\sqrt{2}} & & & \\
& \frac{\epsilon}{\sqrt{2}} \frac{\epsilon}{\sqrt{2}} & & \\
& \frac{-\epsilon}{\sqrt{2}} \frac{\epsilon}{\sqrt{2}} & & & & \\
& & \frac{\epsilon}{\sqrt{2}} \frac{\epsilon}{\sqrt{2}} & & \\
& & \frac{-\epsilon}{\sqrt{2}} \frac{\epsilon}{\sqrt{2}} & & \\
& & & \frac{\epsilon}{\sqrt{2}} & \frac{\epsilon}{\sqrt{2}} \\
& & & & \frac{-\epsilon}{\sqrt{2}} & \frac{\epsilon}{\sqrt{2}}
\end{array}\right]
$$

are topologically similar but not linearly similar. (They have difference traces.)

We also obtain counterexamples to the topological analogue of de Rham's theorem on differentiable similarity of isometries of $S^{n-1}$.

THEOREM. The isometries of $S^{9}$ given by $\left[\begin{array}{cc}A+1 & 0 \\ 0 & 1\end{array}\right]$ and $\left[\begin{array}{cc}A-1 & 0 \\ O^{2} & 1\end{array}\right]$ are topologically similar but not smoothly or linearly similar.

The topological similarity can be taken to be a diffeomorphism outside of at most a circle in $S^{9}$.

Thus, there is no full analogue in higher dimensions to Poincaré's notion of rotation number.

These conjectures were reformulated in terms of the topological classification of finite dimensional representations of finite groups. Two linear representations $\alpha: G \times R^{n} \rightarrow R^{n}$ and $\beta: G \times R^{n} \rightarrow R^{n}$ of the finite group $G$ are said to be topologically equivalent if there is a homeomorphism $f: R^{n} \rightarrow R^{n}$ with $^{2} f(0)=0$ and $\alpha(g, f(x))=f(\beta(g, x)), g \in G, x \in R^{n}$. The conjectures thus stated that for any cyclic group (and hence for any group) topological equivalence of representations implied linear equivalence. The results of Kuiper and Robbins [5], Sullivan, and Schultz [9] show that this conjecture is valid for 
cyclic groups of order $1,2,4, p^{s}, 2 p^{s}, p$ an odd prime (and hence for any group with elements only of those orders) and perhaps for some other cases. However, we have:

\section{THEOREM. Let $G$ be a cyclic group of order $4 n, n>1$. Then there are} linearly inequivalent finite dimensional representations of $G$ which are topologically equivalent.

These counterexamples follow from viewing the representations as giving actions of groups on the sphere of unit radius and using our methods for the study of pseudo-free group actions on spheres; pseudo-free group actions of a finite group are those for which the set of points at which the action is not free is finite. We have studied the classification of such actions quite generally. Results on P. L. classification of cyclic group actions appeared in [2]; the algebraic and geometrical methods developed there are used here.

In particular, for the matrices $A_{\epsilon}, \epsilon= \pm 1$ described above, the quotient spaces $Q_{\epsilon}$ of the actions on the unit sphere are the one-point compactifications of the total space of nontrivial line bundles over lens spaces. These spaces $Q_{+1}$ and $Q_{-1}$ are not manifolds in a neighborhood of the compactification points. It can be shown, using Reidmeister torsion that they are not homeomorphic; however, we construct " $h$-cobordisms" between them and then conclude that $Q_{+1} \times$ $R$ is homeomorphic to $Q_{-1} \times R$ and from this get the first counterexample.

An application of this, in another direction, is that it leads to the first examples of cyclic groups, for example $Z_{8}$, acting smoothly on spheres with the linear representations at the two fixed points of the action being inequivalent They are, however, topologically equivalent.

\section{REFERENCES}

1. V. I. Arnold, Ordinary differential equations, M.I.T. Press, 1973.

2. S. E. Cappell and Julius L. Shaneson, Pseudo-free group actions. I, Proceedings of the 1978 Aarhus Topology Conference (to appear).

3. - Which groups have pseudo-free actions on spheres (to appear).

4. - Linear representations which are topologically the same (to appear).

5. N. Kuiper and J. W. Robbin, Topological classification of linear endomorphisms, Invent. Math. 19 (1973), 83-106.

6. H. Poincaré, Sur les courbes définies par les équations différentielles, Oeuvres de H. Poincaré, Vol. I 1928, Gauthier Villars, Paris.

7. G. de Rham, Reidemeister's torsion invariant and rotations of $\boldsymbol{S}^{\boldsymbol{n}}$, International Conf. on Differential Analysis, pp. 27-36, Oxford University Press, 1964.

8. G. de Rham, S. Maumaury and M. Kervaire, Torsion et type simple d'homotopie, Lecture Notes in Math., vol. 48, Springer-Verlag, Berlin and New York, 1967.

9. R. Schultz, On the topological classification of linear representations (to appear).

COURANT INSTITUTE OF MATHEMATICAL SCIENCES, NEW YORK UNIVERSITY, NEW YORK 10012 